\title{
Performance of the ATLAS electromagnetic calorimeter under beam tests
}

\author{
Fabrice Hubaut ${ }^{\text {a * }}$
}

\author{
${ }^{a}$ CPPM, CNRS/IN2P3, Univ. Méditerranée, Marseille, France
}

The physics program at LHC is highly demanding in terms of detector performance. In particular, the ATLAS electromagnetic calorimeter has to match challenging requirements for energy, position and time resolutions. Calorimeter prototype and production modules have been tested under electron beams at CERN during the last three years. Results are presented and compared to ATLAS requirements.

\section{Introduction}

The ATLAS (A Toroidal LHC ApparatuS) experiment [1], presently under construction, will start operation in 2007 at the LHC [2] protonproton collider at CERN. This multi-purpose detector has a wide physics program, spanning from precision measurements of $\mathrm{W}^{ \pm}$bosons, top and bottom quarks properties, to Higgs boson or supersymmetric particle searches. In most cases, the electromagnetic (EM) calorimeter will play a key role in measuring energy, position and time of electrons and photons.

\section{General layout of the ATLAS electro- magnetic calorimeter}

The LHC extreme operating conditions impose severe constraints on detectors, in terms of radiation tolerance, background rejection capability, noise handling, response speed, spatial coverage and time stability. The EM calorimeter is a lead-liquid argon (LAr) sampling calorimeter with an accordion geometry [3], that guarantees a full azimuthal coverage. It is divided in one barrel $(|\eta|<1.475)$ and two end-caps $(1.375<|\eta|<3.2)$ and is segmented in depth in three compartments (see figure 1). The sampling 1 (front) is made of narrow strips and performs precise position measurements and $\gamma / \pi^{0}$ separation. The sampling 2 (middle) has a depth of 16 to $18 \mathrm{X}_{0}$ and collects most of the $\mathrm{e} / \gamma$ shower energy. The sampling 3 (back) recovers high energy tails and helps to separate hadronic to elec-

${ }^{*}$ On behalf of the ATLAS liquid argon group. tromagnetic particles. In addition, a thin presampler detector corrects energy losses in the upstream material for $|\eta|<1.8$. In total, almost 200,000 read-out channels give the detector a high granularity. Liquid argon has been chosen for its intrinsic linear behavior, response stability and radiation tolerance. For ease of construction, the barrel part is divided in 32 modules and each end-cap wheel is made of 8 modules. The construction, test and integration of these modules are presently well advanced, and are detailed in a separate contribution [4] within this publication.

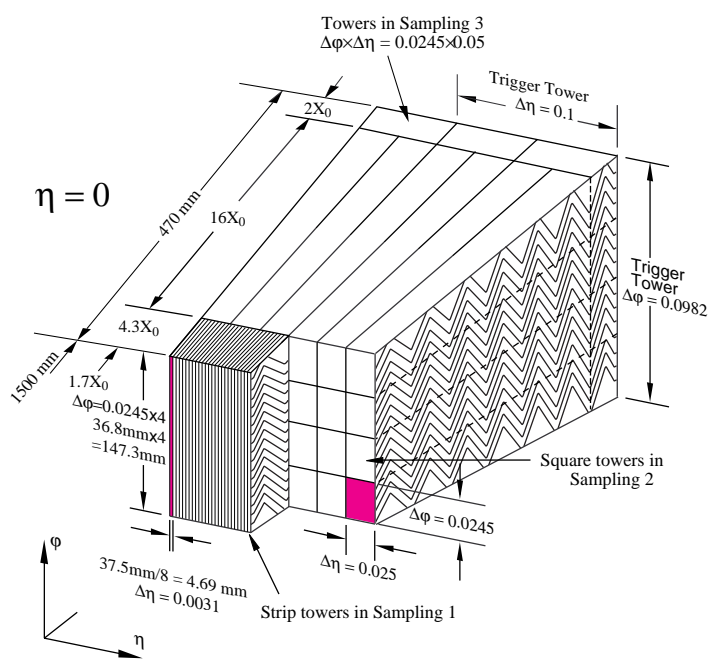

Figure 1. Sketch of the accordion structure and sampling segmentation of the EM calorimeter. 

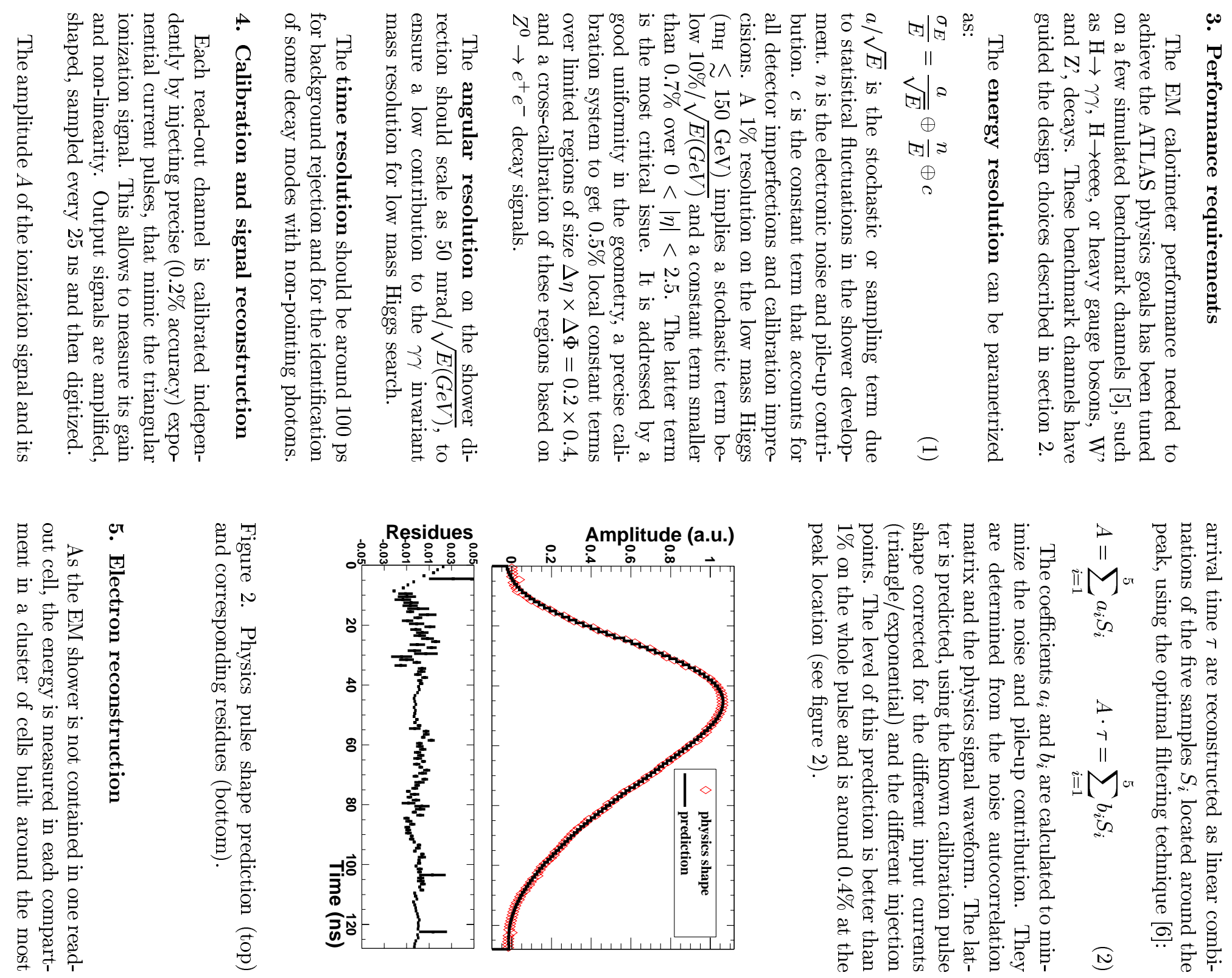
energetic one. In order to compensate for energy losses in front of the calorimeter and leakage beyond it, weights are applied to the presampler and the back compartment responses. Moreover, the finite size of the cluster causes a lateral leakage affecting the energy measurement at a level below $0.4 \%$, and the accordion geometry induces a modulation along $\Phi$ with similar magnitude. In addition, the specific setting of the high voltage by finite sectors in the end-cap induces a linear variation of the energy response as a function of $\eta$ in each sector. Finally, unlike in ATLAS, beam test particle arrival times are asynchronous with respect to the $40 \mathrm{MHz}$ clock, and the energy measurement is sensitive to this phase. All these effects are found in good agreement with simulations and have been corrected for.

\section{Beam test results}

The performance of the EM calorimeter has been extensively tested under electron beams using two full-size prototype modules (one for the barrel and one for the end-cap) and seven production modules (four barrel and three endcap). An ATLAS-like electronics was used. Results from prototype modules, including noise, cross-talk, time stability, temperature effect, response to muons, $\gamma / \pi^{0}$ separation, are extensively described in $[7,8]$. They allowed to improve calorimeter performance. The following results have been obtained with production modules and are similar for all tested modules.

\subsection{Energy resolution and uniformity}

Energy scans from 10 to $245 \mathrm{GeV}$ have been performed at several positions. After unfolding noise and beam energy uncertainty, the sampling term $a$ is found below $10 \% \sqrt{\mathrm{GeV}}$ (resp. 12.5) for every barrel (resp. end-cap) positions, whereas the local constant term $c$ is everywhere smaller than $0.4 \%$. This is in good agreement with ATLAS specifications (section 3 ). The linearity is found to be better than $\pm 1 \%$.

To estimate the global constant term of equation (1), the local constant term has to be added quadratically with the response non-uniformity.
The latter has been determined by performing position scans through every module cells. As a result, the response non-uniformity is lower than $0.6 \%$ on the whole module for the barrel and the end-cap (see figure 3). It is smaller than $0.5 \%$ in regions of size $\Delta \eta \times \Delta \Phi=0.2 \times 0.4$. As required, the resulting global constant term is below $0.7 \%$.

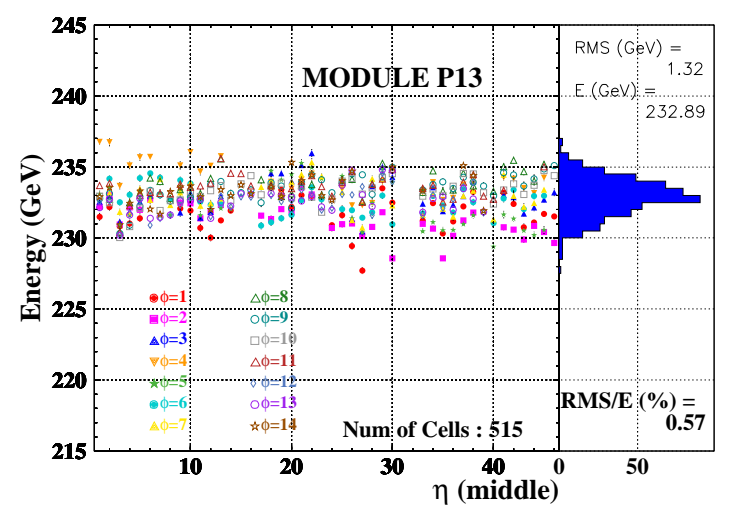

(a)



(b)

Figure 3. Response uniformity of (a) a barrel and (b) an end-cap production module. 


\subsection{Position measurement resolution}

Energy-weighted barycenters are calculated in the front and/or in the middle compartments to perform position measurements. In the $\eta$ direction, corrections for finite cell size are done and the beam chambers resolution is unfolded. The position resolution in the front (resp. middle) section is stable along $\eta$ and amounts to $0.15 \cdot 10^{-3}$ $\eta$-units (resp. $0.35 \cdot 10^{-3}$ ). These two $\eta$ measurements can be combined with the longitudinal shower barycenters to estimate the shower direction. An average preliminary $50 \mathrm{mrad} / \sqrt{E(G e V)}$ resolution is achieved over the whole calorimeter which is in agreement with simulations and within ATLAS specifications.

\subsection{Time measurement resolution}

The optimal filtering technique provides information on the particle arrival time (equation (2)). Cell to cell time differences are studied. Figure 4 shows the results obtained for one barrel cell and its neighbors, as a function of the energy. They are in agreement with the expected electronics contribution. The time resolution amounts to $\sim 70$ ps at $70 \mathrm{GeV}$, which is within ATLAS specifications.

\section{Conclusions and outlook}

The several beam tests performed on prototype and production modules show that the ATLAS EM calorimeter meets the physics specifications. The construction, test and integration of the final modules are presently well proceeding [4]. Combined runs of a full barrel wedge and of all endcap liquid argon calorimeters will take place next year, and will provide a first glimpse of the ATLAS detector.

\section{Acknowledgment}

I thank my ATLAS-Larg-EM colleagues for providing me with figures and results. I am indebted to E. Monnier, P. Pralavorio and L. Serin for discussions in the preparation of the talk and the manuscript.

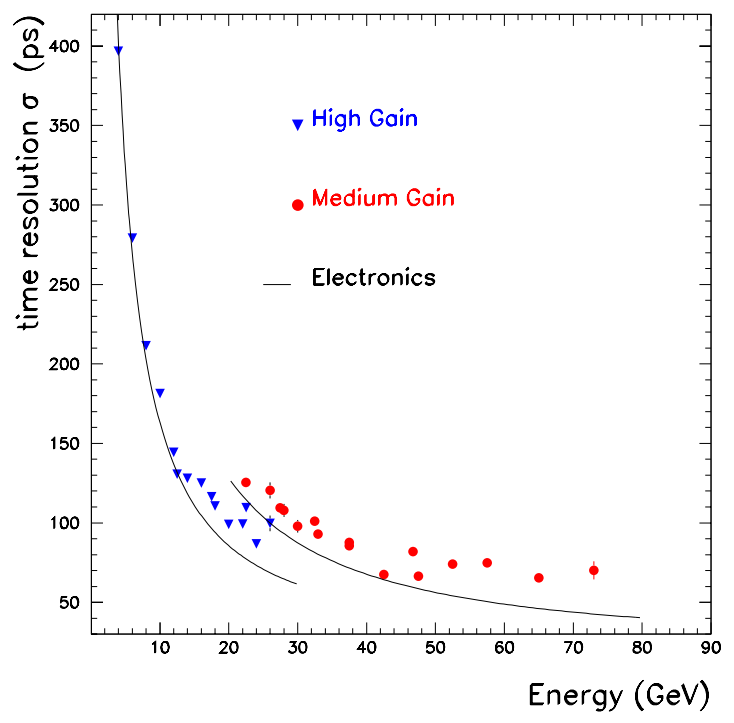

Figure 4. Time resolution (symbols) as a function of the energy, and expected electronics contribution (line).

\section{REFERENCES}

1. The ATLAS Technical Proposal, CERN/LHCC/94-43 (1994).

2. The Large Hadron Collider, CERN/AC/9505 (1995).

3. ATLAS Liquid Argon Calorimeter, Technical Design Report, CERN/LHCC/96-41 (1996).

4. A. Jérémie, The ATLAS liquid argon electromagnetic calorimeter construction status, these proceedings.

5. ATLAS Calorimeter Performance, Technical Design Report, CERN/LHCC/96-40 (1996).

6. W.E. Cleland, E.G. Stern, Nucl. Inst. Meth. A338 (1994) 467.

7. ATLAS Electromagnetic Liquid Argon Calorimeter Group, Performance of the ATLAS electromagnetic calorimeter barrel module 0, Nucl. Inst. Meth. A500 (2003) 202.

8. ATLAS Electromagnetic Liquid Argon Calorimeter Group, Performance of the ATLAS electromagnetic calorimeter end-cap module 0, Nucl. Inst. Meth. A500 (2003) 178. 